OPEN ACCESS

Edited by:

Marc Bramkamp,

Ludwig-Maximilians-Universität

München, Germany

Reviewed by:

Clayton Caswell,

Virginia Tech, United States

Claudio Valverde,

Universidad Nacional de Quilmes

(UNQ), Argentina

${ }^{*}$ Correspondence:

Udo Bläsi

udo.blaesi@univie.ac.at

Specialty section:

This article was submitted to

Microbial Physiology and Metabolism,

a section of the journa

Frontiers in Microbiology

Received: 10 October 2017 Accepted: 08 November 2017 Published: 23 November 2017

Citation:

Tata M, Amman F, Pawar V, Wolfinger MT, Weiss S, Häussler S and Bläsi U (2017) The Anaerobically

Induced sRNA Pail Affects

Denitrification in Pseudomonas

aeruginosa PA14.

Front. Microbiol. 8:2312.

doi: 10.3389/fmicb.2017.02312

\section{The Anaerobically Induced sRNA Pail Affects Denitrification in Pseudomonas aeruginosa PA14}

\author{
Muralidhar Tata ${ }^{1}$, Fabian Amman ${ }^{2}$, Vinay Pawar ${ }^{3,4}$, Michael T. Wolfinger ${ }^{2}$, \\ Siegfried Weiss ${ }^{3}$, Susanne Häussler ${ }^{4,5}$ and Udo Bläsi ${ }^{1 *}$
}

${ }^{1}$ Department of Microbiology, Immunobiology and Genetics, Max F. Perutz Laboratories, University of Vienna, Vienna, Austria, ${ }^{2}$ Institute of Theoretical Chemistry, University of Vienna, Vienna, Austria, ${ }^{3}$ Institute of Immunology, Hannover Medical School, Hannover, Germany, ${ }^{4}$ Department of Molecular Bacteriology, Helmholtz Center for Infection Research, Braunschweig, Germany, ${ }^{5}$ Institute of Molecular Bacteriology, Twincore, Center for Experimental and Clinical Infection Research, Hannover, Germany

Pseudomonas aeruginosa is an opportunistic pathogen that can thrive by anaerobic respiration in the lungs of cystic fibrosis patients using nitrate as terminal electron acceptor. Here, we report the identification and characterization of the small RNA Pail in the $P$. aeruginosa strain 14 (PA14). Pail is anaerobically induced in the presence of nitrate and depends on the two-component system NarXL. Our studies revealed that Pail is required for efficient denitrification affecting the conversion of nitrite to nitric oxide. In the absence of Pail anaerobic growth was impaired on glucose, which can be reconciled with a decreased uptake of the carbon source under these conditions. The importance of Pail for anaerobic growth is further underlined by the observation that a pail deletion mutant was impaired in growth in murine tumors.

Keywords: Pseudomonas aeruginosa, anaerobiosis, small RNA, denitrification, nitrite

\section{INTRODUCTION}

Pseudomonas aeruginosa (Pae) is an opportunistic human pathogen that causes severe infections in immunocompromised individuals, burn patients, and patients suffering from cystic fibrosis (CF). Pae can form biofilms in the lungs of CF patients under microaerobic or anaerobic conditions, underscoring the importance of anaerobic metabolism for pathogenicity (Yoon et al., 2002; Hassett et al., 2009; Kolpen et al., 2014). The switch of Pae from aerobic to anaerobic growth is controlled by the master regulator ANR (Zimmermann et al., 1991), which responds to oxygen limitation through its $[4 \mathrm{Fe}-4 \mathrm{~S}]^{+2}$ cluster (Ye et al., 1995). ANR acts as an upstream activator of the denitrification pathway by stimulating transcription of the nitrate-responsive two component regulatory system NarXL and the nitric oxide-dependent regulator DNR. In conjunction with NirQ, transcription of which is activated by DNR, these transcriptional regulators control a regulatory cascade, which results in the expression of genes encoding enzymes required for the reduction of nitrate to dinitrogen in four consecutive steps (Supplementary Figure S1; Schreiber et al., 2007).

During the last decade numerous small non-coding RNAs (sRNAs) have been identified in different pathogens, such as Escherichia coli, Listeria monocytogenes, Staphylococcus aureus, Vibrio cholera, and Pae (Gottesman and Storz, 2011; Caldelari et al., 2013). In general, bacterial sRNAs are induced by different environmental cues and their role in post-transcriptional regulation permits a fast adaptation to stressors and/or habitats (Hoe et al., 2013). The two major mechanisms by which sRNAs modulate gene expression includes base-pairing with target mRNAs or sequestration 
of a regulatory protein (Storz et al., 2011). At least in Enterobacteriaceae it is well-established that base-pairing sRNAs often require the RNA chaperone $\mathrm{Hfq}$. Hfq fulfills several functions in post-transcriptional regulation. It can stabilize small regulatory RNAs (sRNAs) and facilitate annealing between sRNAs and their target mRNAs. The latter mode of action may result either in translational repression accompanied by degradation of both RNAs or in translational activation and consequently in stabilization of the mRNA (Vogel and Luisi, 2011).

Although many regulatory RNA candidates have been identified in different Pae strains (Livny et al., 2006; Sonnleitner and Haas, 2011; Ferrara et al., 2012; Wurtzel et al., 2012), only a few of them such as RsmW/RsmY/RsmZ, CrcZ, PhrS, NrsZ, and EsrA have been functionally characterized. The RNAs CrcZ (Sonnleitner and Bläsi, 2014) and RsmW/RsmY/RsmZ (Lapouge et al., 2008; Miller et al., 2016) function by sequestering regulatory proteins. On the other hand, the bona fide sRNAs PhrS (Sonnleitner et al., 2011), PrrF1-2 (Reinhart et al., 2015), NrsZ (Wenner et al., 2014), and EsrA (Ferrara et al., 2015) basepair with and regulate their cognate target mRNAs. The Pae O1 sRNA PhrS, which has been implicated in the synthesis of the Pseudomonas quinolone signal (PQS), was hitherto the only identified sRNA whose expression depends on the anaerobic regulator ANR (Sonnleitner et al., 2011). In E. coli, the sRNA FnrS has been reported to be induced under hypoxic conditions in an FNR dependent manner. It was shown to regulate at least 32 mRNAs, many of which encode enzymes involved in central and energy metabolism, while a few encode enzymes required for aerobic respiration (Boysen et al., 2010; Durand and Storz, 2010). Hence, Durand and Storz (2010) suggested that FnrS serves to increase the efficiency of anaerobic metabolism by repressing functions that are not required under these conditions.

Given the importance of anaerobic biofilms in Pae pathogenicity, in this study we aimed at identifying novel regulatory sRNAs induced during anaerobiosis. We first reanalyzed a RNAseq based transcriptome study performed with the clinical isolate $P$. aeruginosa 14 (PA14) (Tata et al., 2016) for sRNA candidates that are induced at $30 \mathrm{~min}$ and $96 \mathrm{~h}$ after oxygen depletion, respectively. Among already known and new differentially abundant sRNA candidates this survey revealed the putative sRNA PA14_13970.1, which we termed PaiI. We show that Pail transcription depends on the two component system NarXL. Further studies revealed that PaiI is required for optimal anaerobic growth on glucose and that it is required for a rapid conversion of $\mathrm{NO}_{2}$ to NO. A PA14 paiI deletion mutant showed an increased accumulation of nitrite and decreased nitrite reductase activity. Over-production of the transcriptional activator DNR rescued the paiI deletion phenotype. However, neither PaiI nor DNR over-production affected the levels of the nitrite reductase which suggests that PaiI indirectly impacts on denitrification. The importance of Pail for anaerobic growth was further underlined by the observation that a paiI deletion mutant was impaired in growth in transplanted murine tumors that consist of hypoxic/anaerobic areas.

\section{MATERIALS AND METHODS}

\section{Bacterial Strains and Growth Conditions}

Strains and plasmids used in this study are listed in Supplementary Table S1. Unless indicated otherwise, the Pae strains were grown at $37^{\circ} \mathrm{C}$ in basal salt medium (BSM) (Durham and Phibbs, 1982) supplemented with glucose or other carbon sources specified in the text (final concentration $20 \mathrm{mM}$ ). Anoxic growth was performed in an anaerobic chamber $(95 \%$ $\mathrm{N}_{2}, 5 \% \mathrm{H}_{2}$ ). For growth under anoxic conditions the medium was supplemented with $100 \mathrm{mM} \mathrm{KNO}_{3}$. The E. coli strain $\mathrm{DH} 5 \alpha$ was used for the construction of plasmids. When required, the following concentrations of antibiotics were used: $15 \mu \mathrm{g} / \mathrm{ml}$ gentamycin, $100 \mu \mathrm{g} / \mathrm{ml}$ ampicillin, and $25 \mu \mathrm{g} / \mathrm{ml}$ tetracycline for E. coli; $50 \mu \mathrm{g} / \mathrm{ml}$ gentamycin, $250 \mu \mathrm{g} / \mathrm{ml}$ carbenicillin and $100 \mu \mathrm{g} / \mathrm{ml}$ of tetracycline for Pae.

\section{Construction of PA14 $\Delta$ pail and PA14_narL-pail}

A markerless in-frame deletion of the paiI gene was constructed by homologous recombination (Ye et al., 1995). The PA14 genome coordinates referred to below are taken from http://www.pseudomonas.com/. First, the 717 bp upstream (PA14 genome coordinates: 1.198.245-1.198.961) and $700 \mathrm{bp}$ downstream sequences (PA14 genome coordinates: 1.199.0481.199.747) of the paiI gene were PCR-amplified using the primer pairs C99/E99 and D99/F99 (Supplementary Table S2), respectively. The PCR products were then linked by an overlap PCR to generate a fragment containing the in-frame deletion in paiI. The linked product was inserted into the KpnI and XbaI sites of plasmid pME3087 (Supplementary Table S1). The paiI deletion (PA14 genome coordinates: 1.198.962-1.199.047) was verified by DNA sequencing. The corresponding plasmid was then transformed into PA14 with the aid of E. coli strain HB101 (pRK2013). Single-crossover and double-crossover mutants were selected based on tetracycline resistance and sensitivity (Ye et al., 1995). The paiI gene deletion was confirmed by means of PCR.

Deletion of the NarL binding site within the pail promoter sequence (PA14 genome coordinates: 1.199.107-1.199.115) was performed as described above. The primer pairs C99/P105 and D99/Q105 (Supplementary Table S2) were used for amplification of the upstream (1.198.245-1.199.106) and downstream (1.199.116-1.199.747) sequences of the NarL binding motif, respectively. The deletion of the NarL binding site within the paiI promoter in strain PA14 narL-paiI was verified by means of PCR and subsequent DNA sequencing.

\section{Construction of Plasmids}

DNA cloning and plasmid preparations were performed according to standard methods (Sambrook and Russell, 2001). The paiI expression plasmid pME-paiI was generated by PCR amplification of the paiI gene using PA14 genomic DNA as template and primers P107 and H99 (Supplementary Table S2). The PCR products were cleaved with SmaI and PstI and then ligated into the corresponding sites of plasmid pME4510-1 (Supplementary Table S1). Plasmid pME4510-1 was derived from 
plasmid pME4510 (Rist and Kertesz, 1998), wherein the lacI ${ }^{Q}$ $\mathrm{P}_{\text {tac }}$ region was $\mathrm{PCR}$ amplified from plasmid pMMBH67HE (Fürste et al., 1986) using the oligonucleotides Q107 and L85 (Supplementary Table S2). The resulting PCR products were then digested with SmaI and EcoRI and ligated into the corresponding sites of pME4510 to generate pME4510-1

The plasmids pMMB-anr, pMMB- $d n r$, and pMMB-nirQ were constructed by means of PCR amplification of the respective genes using PA14 genomic DNA as template and the primers listed in Supplementary Table S2. The PCR products were cleaved with HindIII and BamHI, and then ligated into the corresponding sites of vector $\mathrm{pMMB} \Delta$ rbs (Sonnleitner and Bläsi, 2014). The forward primers used for PCR amplification of either gene comprised a ribosome binding site (see Supplementary Table S2).

\section{Northern-Blot Analysis}

Total RNA was isolated from cultures using the TRIzol Reagent (Ambion) according to the manufacturer's instructions. For Northern-blot analysis, $10 \mu \mathrm{g}$ of total RNA were heated at $65^{\circ} \mathrm{C}$ for $5 \mathrm{~min}$ in loading buffer ( $5 \mathrm{mM}$ EDTA, $0.025 \%$ xylene cyanol, $0.025 \%$ bromophenol blue dissolved in formamide) and resolved on $8 \%$ polyacrylamide/ $8 \mathrm{M}$ urea gels. The RNA was transferred onto Hybond $\mathrm{N}+$ nylon membranes (GEHealthcare) using a semi-dry electroblotting apparatus (Transblot SD cell, BioRad) set at $13 \mathrm{~V}$ for $45 \mathrm{~min}$ followed by UV-cross-linking. A DNA oligonucleotide probe specific for PaiI (Supplementary Table S2) was $5^{\prime}$-end labeled with $\left[\gamma^{-32} \mathrm{P}\right]$ ATP using T4 polynucleotide kinase. The $\left[{ }^{32} \mathrm{P}\right]$-labeled oligonucleotide was heated at $95^{\circ} \mathrm{C}$ for $2 \mathrm{~min}$, added to pre-hybridized membranes and incubated at $52^{\circ} \mathrm{C}$ overnight. Pre-hybridization and hybridization were performed in Roti ${ }^{\circledR}$ Hybrid Quick buffer (Carl Roth, Karlsruhe, Germany) supplemented with $0.1 \mu \mathrm{g} / \mathrm{ml}$ salmon sperm DNA. A 5S rRNA-specific oligonucleotide was used as a loading control, (Supplementary Table S2). The signals were visualized using a PhosphorImager (Molecular Dynamics).

\section{In Vitro Transcription}

For in vitro transcription of paiI (126 nt) and paiI-1 (141 nt: used for sequencing reactions in primer extension analyses), the AmpliScribe T7-Flash Transcription Kit (Epicentre Biotechnologies) was used according to the manufacturer's instructions. PCR fragments used for in vitro transcription were generated with the primer pairs H109/I86 paiI (126 nt) and Q106/I86 paiI-1 (141 nt) using PA14 genomic DNA as template (Supplementary Table S2). The forward primers contained a T7 promoter sequence.

\section{Primer Extension Analysis}

The primer extension analysis was performed as described (Ausubel et al., 1997). Total RNA from B-96 cultures was prepared using the TRIzol Reagent (Ambion) according to the manufacturer's instructions. Primer R105 (Supplementary Table S2) was $5^{\prime}$-end labeled with $\left[\gamma^{32} \mathrm{P}\right]$ ATP using T4 polynucleotide kinase (Thermo Fisher Scientific) and annealed to $10 \mu \mathrm{g}$ of total RNA. Primer extension reactions were carried out with avian myeloblastosis virus (AMV) reverse transcriptase
(Promega). The extension products were loaded onto a $8 \mathrm{M}$ urea- $8 \%$ polyacrylamide gel next to the sequencing reactions performed with the same primer using in-vitro transcribed Pail-1 (141 nt) sRNA as template.

\section{Enzymatic Probing}

Secondary structure probing of the sRNA PaiI was carried out as described (Franch et al., 1999). Briefly, 0.05 pmol of $5^{\prime}$ end${ }^{32} \mathrm{P}$ labeled in vitro transcribed PaiI RNA was denatured for $3 \mathrm{~min}$ at $85^{\circ} \mathrm{C}$ and slowly cooled to room temperature. Then, the RNA was incubated for 0,10 , and $15 \mathrm{~min}$ in reaction buffer ( $10 \mathrm{mM}$ Tris, $60 \mathrm{mM} \mathrm{NH}_{4} \mathrm{Cl}, 6 \mathrm{mM} \beta$-mercaptoethanol, $2 \mathrm{mM}$ magnesium acetate, $\mathrm{pH}$ 7.4) containing $0.2 \mathrm{U}$ of RNase T1 (Sigma-Aldrich). The alkaline ladder was generated by incubation of 0.05 pmol $5^{\prime}$ end-labeled RNA at $85^{\circ} \mathrm{C}$ for $3 \mathrm{~min}$ in reaction buffer $\left(10 \mathrm{mM}\right.$ Tris, $60 \mathrm{mM} \mathrm{NH}_{4} \mathrm{Cl}, 6 \mathrm{mM}, \beta$ mercaptoethanol, $2 \mathrm{mM}$ Magnesium acetate, $\mathrm{pH}$ 7.4) containing $35 \mathrm{mM} \mathrm{NaHCO} 3$ (pH 9.0). The reactions were stopped by adding equal volumes of loading dye (5 mM EDTA, $0.025 \%$ xylene cyanol, $0.025 \%$ bromophenol blue) and analyzed on $8 \mathrm{M}$ urea-8\% polyacrylamide gels. The signals were visualized using a PhosphorImager (Molecular Dynamics).

\section{Pail Synthesis and Stability}

To monitor transcription of paiI after shift from aerobic to anaerobic conditions, PA14 was first grown aerobically in SCFM (Palmer et al., 2007) supplemented with $100 \mu \mathrm{M} \mathrm{FeSO}_{4}$ to an $\mathrm{OD}_{600}$ of 0.45 . Then $\mathrm{KNO}_{3}$ was added to a final concentration of $100 \mathrm{mM}$, followed by incubation under anaerobic conditions. Total RNA was isolated at times 15, 30, 60, and 120 min to detect the Pail levels by Northern-blotting as described above.

To measure the stability of Pail after shift from anaerobic to aerobic conditions, PA14 was first grown in $50 \mathrm{ml}$ flasks for $96 \mathrm{~h}$ under anaerobic conditions in SCFM in the presence of $100 \mathrm{mM}$ $\mathrm{KNO}_{3}$ and $100 \mu \mathrm{M} \mathrm{FeSO}_{4}$ without shaking. Then, the cultivation was continued with shaking under aerobic conditions. Total RNA was isolated at times 15, 30, 60, and 120 min after the shift and PaiI was detected by Northern-blotting as described above.

\section{Determination of the CFU}

Overnight cultures of PA14 were inoculated in fresh BSM medium supplemented with $20 \mathrm{mM}$ glucose at an initial $\mathrm{OD}_{600}$ of 0.05. Cultures were grown aerobically to an $\mathrm{OD}_{600}$ of 0.45 , at which, $\mathrm{KNO}_{3}$ (100 $\mathrm{mM}$ final concentration) was added. The cultures were then transferred to the anaerobic chamber. A sample of each culture was withdrawn before the shift $(0 \mathrm{~h})$ and after 2, 4, 6, and $8 \mathrm{~h}$. The samples were serially diluted and plated on LB agar plates. The plates were incubated overnight aerobically at $37^{\circ} \mathrm{C}$ to determine the CFU. All experiments were performed in triplicate and the results are presented as mean \pm standard deviation (SD).

\section{Glucose Uptake}

The glucose concentration present in the supernatant of the cultures was measured by using the glucose (GO) assay kit (Sigma-Aldrich: GAGO20-1KT). The cultures were grown under the same conditions as used for determination of the CFU. The 
cultures were then transferred to the anaerobic chamber. One milliliter sample of each culture was withdrawn before the shift $(0 \mathrm{~h})$ and 1 and $2 \mathrm{~h}$ thereafter. The samples were centrifuged at $4,000 \mathrm{rpm}$ in a benchtop centrifuge for $10 \mathrm{~min}$. The supernatant was filtered through a $0.2 \mu \mathrm{m}$ filter and used for determination of the glucose concentrations. The amount of glucose present in the supernatant is inversely proportional to the amount of glucose taken up by the cultures. All experiments were performed in triplicates and the results are presented as mean \pm standard deviation (SD).

\section{Determination of the Nitrite Concentration}

The nitrite concentration in the supernatants of cultures was determined by the Griess assay (Nicholas and Nason, 1957). The cultures were grown under the same conditions as used for determination of the CFU. At different times, $1 \mathrm{ml}$ of the cultures was centrifuged at $4,000 \mathrm{rpm}$ in a benchtop centrifuge for $10 \mathrm{~min}$. Equal amounts of $1 \% \mathrm{w} / \mathrm{v}$ sulfanilic acid dissolved in $20 \% \mathrm{HCl}$ and $1.3 \mathrm{mg} / \mathrm{ml}$ naphthylenediamine dihydrochloride (NEDD) solutions were added to the supernatant. The reaction mixture was incubated for $5 \mathrm{~min}$ at room temperature to allow formation of the red azo dye. The absorbance was measured at $540 \mathrm{~nm}$, and the nitrite concentration was determined by comparing the values to a standard curve obtained with different nitrite concentrations. All experiments were performed in triplicates and results are presented as mean \pm standard deviation (SD).

\section{Determination of the Nitrate Reductase Activity}

The nitrate reductase activity was determined as described previously (Stewart and Parales, 1988). The cultures were grown under the same conditions as used for determination of the CFU. At the different time points, $1 \mathrm{ml}$ samples were collected and centrifuged at $4000 \mathrm{rpm}$ in a benchtop centrifuge for $10 \mathrm{~min}$. The cell pellet was washed twice with $50 \mathrm{mM}$ phosphate buffer, $\mathrm{pH} 7.2$, and resuspended in $1 \mathrm{ml}$ of the same buffer. The cells were permeabilized by addition of $50 \mu \mathrm{l}$ toluene followed by shaking for $1 \mathrm{~h}$ at $400 \mathrm{rpm}$ at $37^{\circ} \mathrm{C} .800 \mu \mathrm{l}$ of permeabilized cells were mixed with $100 \mu \mathrm{l}$ of freshly prepared $0.5 \mathrm{mg} / \mathrm{ml}$ methyl viologen solution. Nitrate reduction was initiated by adding $100 \mu \mathrm{l}$ of a solution containing $4 \mathrm{mg} / \mathrm{ml}$ sodium dithionite, 4 $\mathrm{mg} / \mathrm{ml}$ sodium bicarbonate, and $100 \mathrm{mM} \mathrm{KNO}_{3}$. After $30 \mathrm{~min}$ of incubation, the reactions were stopped by vigorous shaking until the dark blue color disappeared. The amount of nitrite formed in the reaction mixture was measured by using the Griess assay (Nicholas and Nason, 1957). Enzyme activity is defined as the amount of nitrate reductase required to produce $1 \mathrm{nmol}$ nitrite $\mathrm{min}^{-1} \mathrm{mg}^{-1}$ protein. All experiments were performed in triplicates and the results are presented as mean \pm standard deviation (SD).

\section{Determination of the Nitrite Reductase Activity}

The nitrite reductase activity was determined by measuring the reduction of $\mathrm{KNO}_{2}$ by methyl viologen (Moir et al., 1993). Sample preparation and processing was performed as specified above for the determination of the nitrate reductase activity. Nitrite reduction was initiated by adding $100 \mu \mathrm{l}$ of a solution containing $4 \mathrm{mg} / \mathrm{ml}$ sodium dithionite, $4 \mathrm{mg} / \mathrm{ml}$ sodium bicarbonate and $200 \mu \mathrm{M} \mathrm{KNO}_{2}$. After $30 \mathrm{~min}$, the assay was stopped by vigorous shaking until the dark blue color disappeared. The amount of nitrite remaining in the reaction mixture was measured by using the Griess assay (Nicholas and Nason, 1957), and was quantified by comparing the values to a standard curve obtained with nitrite. All experiments were performed in triplicates and repeated at least three times. All results are presented as mean \pm standard deviation (SD).

\section{RNA $A_{\text {Seq }}$ Library Construction and Sequence Analysis}

Total RNA was prepared from three biological replicates of PA14 and PA14 $\Delta$ paiI, respectively. The overnight cultures were inoculated in fresh BSM medium supplemented with $20 \mathrm{mM}$ glucose at an initial $\mathrm{OD}_{600}$ of 0.05 . PA14 and PA14 $\Delta$ paiI were first grown aerobically to an $\mathrm{OD}_{600}$ of 0.4 , at which $\mathrm{KNO}_{3}$ (final concentration $100 \mathrm{mM}$ ) was added and then transferred to an anaerobic chamber. After $1 \mathrm{~h}$ of incubation, the cells were harvested for total RNA preparation using the TRIzol Reagent (Ambion) according to the manufacturer's instructions. Under these conditions anaerobiosis was ensured by employing an anaerobic indicator strip (Oxoid). The samples were DNase I treated, followed by phenol-chloroform-isoamyl alcohol extraction and ethanol precipitation. The Ribo-Zero ${ }^{\mathrm{TM}}$ rRNA Removal Kit (Illumina) was used to deplete rRNA from total RNA samples. Libraries were constructed using the NEBNext1Ultra ${ }^{\mathrm{TM}}$ Directional RNA Library Prep Kit from Illumina. $100 \mathrm{bp}$ single end sequence reads were generated using the Illumina HiSeq 2000 platform at the Vienna Biocenter Core Facilities (http://www.csf.ac.at). The sequence reads were adaptor clipped and quality trimmed with trimmomatic (Bolger et al., 2014) with default parameters. Mapping of the samples against the UCBPP-PA14 reference genome (accession number NC_008463) was performed with Segemehl (Hoffmann et al., 2009 , 2014). The uniquely mapped sequencing data were split by strand and further processed for automatic UCSC Genome Browser visualization with the ViennaNGS toolbox (Kent et al., 2002; Wolfinger et al., 2015). Read counting for subsequent DESeq2 (Love et al., 2014) differential gene expression analysis was performed with BEDtools (Quinlan and Hall, 2010). The raw sequencing reads were deposited in the European Nucleotide Archive as a study under the accession number PRJEB22593 (http://www.ebi.ac.uk/ena/data/view/PRJEB22593).

\section{Infection of Tumor-Bearing Mice}

The mouse experiments were performed as previously described (Pawar et al., 2015). Briefly, 7- to 8-week -old female BALB/c mice (Janvier, Germany) were injected intradermally with $5 \times 10^{5}$ cells of the colon carcinoma cell line CT26. Mice bearing tumors of $150-200 \mathrm{~mm}^{3}$ diameter were infected intravenously (i.v.) with 5 $\times 10^{6}$ colony forming units (CFU) of the PA14 strains suspended in phosphate-buffered saline (PBS). All animal experiments were carried out with the permission of the lower Saxony authorities (LAVES-permission No. 33.9-42502-04-12/0713). 
At the indicated times, the mice were sacrificed and the tumors were homogenized in $2 \mathrm{ml}$ of ice-cold $0.1 \%$ (v/v) Triton X100/PBS using gentle MACS ${ }^{\mathrm{TM}}$ M-tubes and a dissociator from Miltenyi Biotec. The samples were serially diluted and plated on Luria-Bertani (LB) agar plates containing ampicillin $(0.1 \mathrm{mg} / \mathrm{ml})$.

\section{Western-Blot Analysis}

The cultures were grown aerobically to an $\mathrm{OD}_{600}$ of 0.45 in minimal medium supplemented with $20 \mathrm{mM}$ glucose followed by addition of KNO3 to a final concentration of $100 \mathrm{mM}$. The cultures were then transferred to the anaerobic chamber. One milliliter sample of each culture was withdrawn after 1 and $2 \mathrm{~h}$. The samples were centrifuged at 4,000 rpm in a benchtop centrifuge for $10 \mathrm{~min}$. Equal amounts of proteins were separated on a $12 \%$ SDS-polyacrylamide gel. The proteins were blotted onto a nitrocellulose membrane, which was blocked with $5 \%$ dried skimmed milk in TBS buffer. The membranes were probed with mouse anti-NirS (Nicke et al., 2013) and rabbit anti-S2 (laboratory stock) antibodies. The antibody-antigen complexes were visualized with alkaline-phosphatase conjugated secondary antibodies (Sigma) using the chromogenic substrates nitro blue tetrazolium chloride (NBT) and 5-Bromo-4-chloro-3-indolyl phosphate (BCIP).

\section{RESULTS}

\section{Identification of SRNA Candidates in Anoxic Biofilms of PA14}

With the aim to identify novel sRNAs that are up-regulated in anoxic biofilms we revisited a recently published RNA $_{\text {Seq }}$-based transcriptome analysis, wherein the transcriptomes of PA14 grown under planktonic (P), anoxic conditions for $30 \mathrm{~min}$ (A-30) and anoxic biofilm conditions for $96 \mathrm{~h}$ (B-96) were compared (Tata et al., 2016). Based on the criteria that sRNA genes should preferably reside in the intergenic regions and/or in the $5^{\prime}$ or the $3^{\prime}$ UTR of annotated genes, we identified 20 sRNA candidates that are differentially abundant in A-30/B-96 conditions when compared with planktonic conditions. Of these, 11 had already been noticed previously and 9 represented novel sRNA candidates (Figure 1, Supplementary Table S3). Next, the Virtual Footprint tool of the PRODORIC database (http:// www.prodoric.de) was used to identify possible ANR and NarL binding motifs in the promoter regions of the up-regulated sRNAs candidates. Among these sRNA candidates, SPA0115 contains a putative ANR binding site, whereas PA14_13970.1, PA14_36030.1, PA14_47800.1, SPA0147, and SPA0011 contain putative NarL signatures (Supplementary Table S3). Moreover, a co-immunoprecipitation experiment with Hfq-specific antibodies (Pusic et al., 2016) indicated that 7 of these 20 sRNA candidates might interact with Hfq (Supplementary Table S3).

\section{Detection and Characterization of the Anaerobically Induced sRNA Pail}

Among all putative sRNAs, the sRNA candidate PA14_13970.1 located in the intergenic region between PA14_13970 and PA14_13990 (http://www.pseudomonas.com/) was most abundant in A-30 and B-96 conditions when compared with

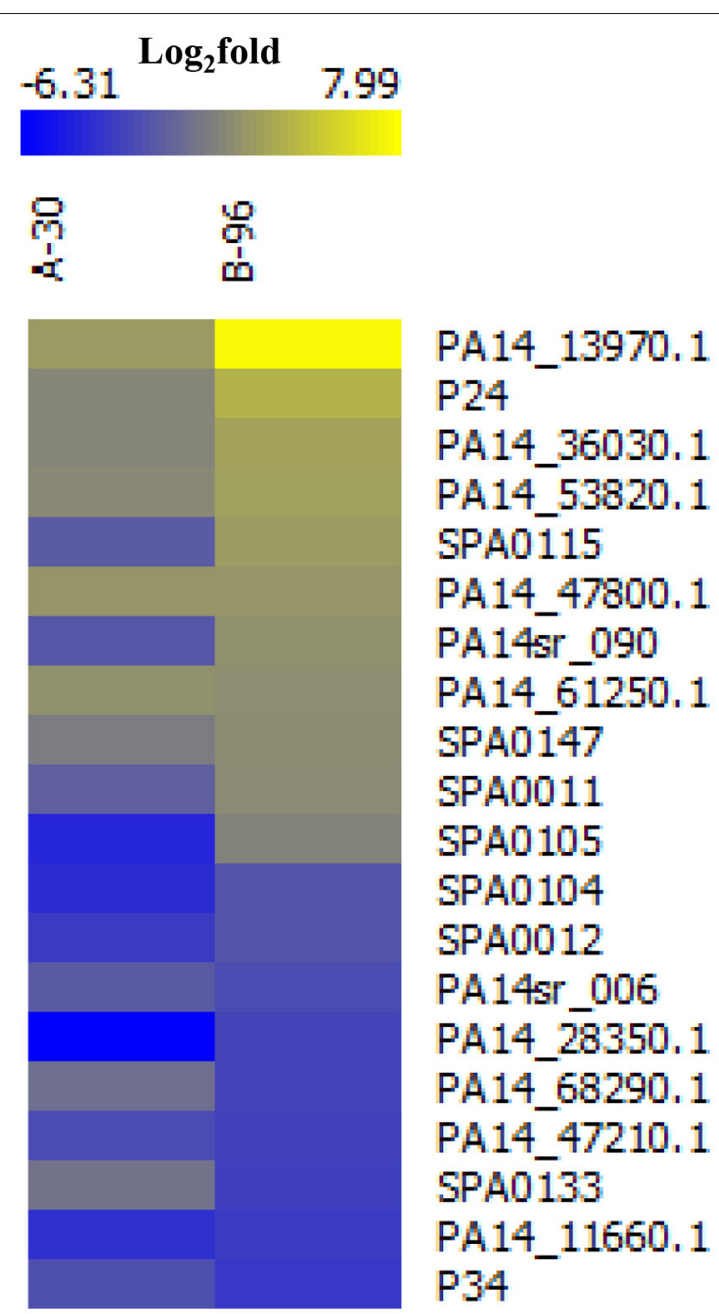

FIGURE 1 | Heat map of differentially abundant novel/annotated sRNAs under the conditions A-30 and B-96 when compared with condition P. The color code shown in the scale at the top denotes log2-fold changes. Blue and yellow indicate a decrease and an increase in the sRNA levels, respectively.

condition P (Figure 1, Supplementary Figure S2A). The putative sRNA was found to be highly conserved among $P$. aeruginosa strains (Zhang et al., 2000), and was termed PaiI (Pseudomonas anaerobically induced RNA $I$ ).

To verify the expression of PaiI under anaerobic conditions total RNA was isolated from PA14 P-cells and B-96 cells (Tata et al., 2016), and examined for the presence of Pail by Northernblotting. As anticipated Pail was exclusively detected under anaerobic conditions in B-96 cells (Figure 2A).

Next, the $5^{\prime}$ end of Pail was mapped by primer extension using total RNA prepared from PA14 B-96 cultures (Supplementary Figure S2B). This analysis indicated that Pail transcription initiates with the $\mathrm{A}$ at nucleotide position 1.199.053 (Supplementary Figure S2C). The transcriptional start site is preceded by a $\sigma^{70}$ recognition motif. Inspection of the secondary structure by RNAfold (rna.tbi.univie.ac.at/cgibin/RNAWebSuite/RNAfold.cgi) predicted a 3'-terminal stem-loop structure followed by a consecutive stretch of five $U$ 
A
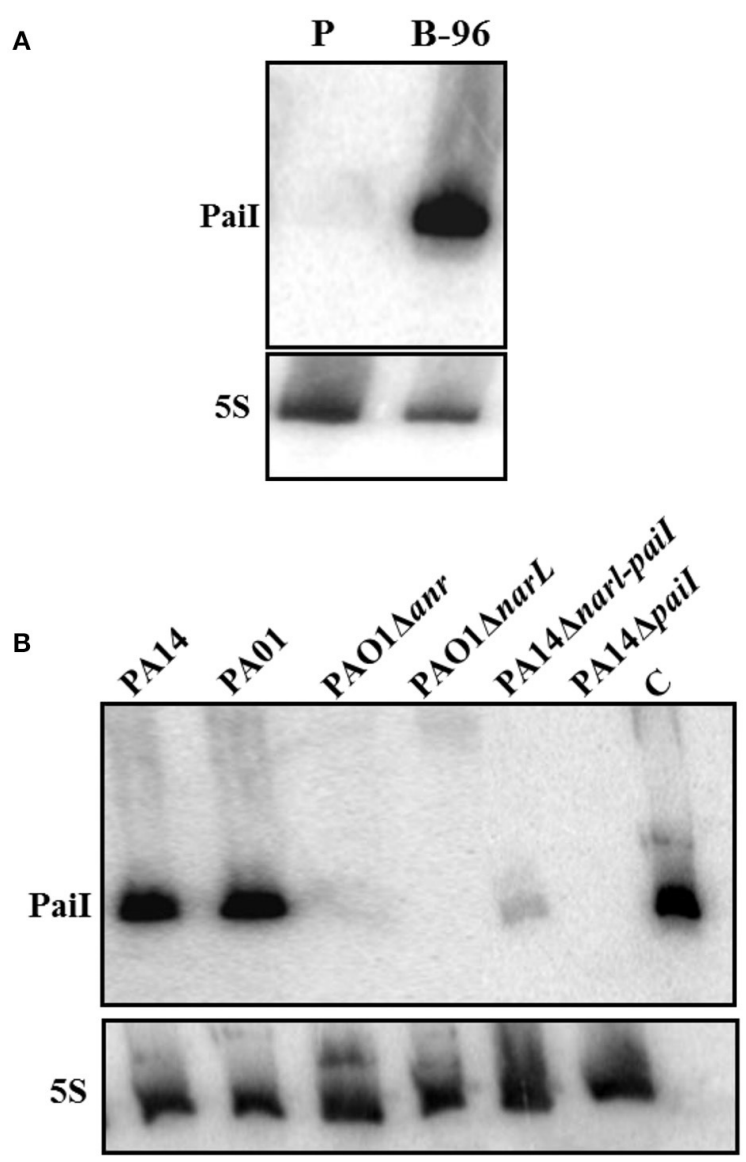

FIGURE 2 | Anaerobic transcription of Pail requires NarXL. (A) Expression of Pail in PA14 B-96 cells. Total RNA was extracted from planktonically growing cells $(P)$ and from anoxic biofilms (B-96) under the same conditions as described (Tata et al., 2016) and the Pail was detected as described in Materials and Methods. (B) The NarXL two-component regulatory system is required for expression of Pail. Detection of Pail by Northern-blotting in total RNA extracted from PAO1 and PA14, and different mutants thereof grown in anoxic biofilms (B-96). $0.6 \mathrm{ng}$ of in vitro transcribed Pail (126 nt) was loaded as a control (C).

residues, indicative for a rho-independent terminator (Farnham and Platt, 1981). With this information, the length of PaiI was calculated with $126 \mathrm{nt}$, which complied with the size judged from Northern-blot experiments (not shown). No extensive secondary structures in addition to the predicted rho-independent terminator (Supplementary Figure S3A) was revealed by employing the RNA-fold algorithm (rna.tbi.univie.ac.at/cgibin/RNAWebSuite/RNAfold.cgi). This finding was supported by in vitro enzymatic probing using RNase T1, which cleaves after $\mathrm{G}$ residues. Although RNase T1 cleavage was not obvious at several positions, cleavage occurred at the majority of $G$ residues preceding the terminal stem-loop structure (Supplementary Figure S3B).

\section{Transcription of Pail Is NarL Dependent}

The primary sequence of PaiI is highly conserved in all sequenced Pae strains (98-100\% identity; Zhang et al., 2000).
In addition, a NarL binding motif (Supplementary Figure S2C) was discerned upstream of a $\sigma^{70}$ promoter for either paiI homolog. Therefore, we first tested the Pail abundance in B-96 cells of $P$. aeruginosa $\mathrm{O} 1$ and in the isogenic mutant strains PAO1 $\Delta a n r$ and PAO1 $\Delta$ narL, respectively. As judged by Northern-blot analyses (Figure 2B) PaiI was absent in the mutant strains, strongly suggesting that transcription is NarL dependent. To verify the NarL dependent transcription of PaiI in PA14, the NarL binding motif within the paiI promoter region (Supplementary Figure S2C) was deleted in strain PA14 $n$ arLpail. When compared with PA14, only minute amounts of Pail were present in B-96 cells of PA14 n narL-paiI (Figure 2B). In addition, PaiI was not detected in B-96 cells when nitrate was omitted from the medium (Supplementary Figure S4). Taken together, these experiments verified that paiI transcription requires the nitrate-responsive two component regulatory system NarXL, transcription of which in turn depends on the anaerobic regulator ANR (Schreiber et al., 2007).

\section{Synthesis and Turnover of Pail}

Next, we studied the time course of induction of paiI after shift from aerobic to anaerobic conditions as described in Materials and Methods. Samples for isolation of total RNA were withdrawn after 15, 30, and $60 \mathrm{~min}$. As shown in Figure 3A, PaiI was detectable after $15 \mathrm{~min}$ and was highly expressed $30 \mathrm{~min}$ after the shift to anaerobiosis. Next, the reverse experiment was performed. B-96 cells were shifted to aerobic conditions with vigorous shaking. As shown in Figures 3B,C, the PaiI levels declined after shift to aerobic conditions. As ANR and NarL are non-functional in the presence of oxygen and in the absence of $\mathrm{KNO}_{3}$, respectively (Schreiber et al., 2007), this observation indicated that Pail is removed when its synthesis discontinues upon shift to aerobiosis.

\section{Pail Is Required for Anoxic Survival of Pae When Growing on Glucose}

As PaiI is only present during anoxic growth, we next asked whether it impacts on growth and viability under these conditions. To address this, a PA14 paiI deletion mutant was constructed, and growth of strain PA14 $\Delta$ paiI in BSM medium supplemented with different carbon sources was compared with that of the wild-type strain PA14. These initial growth experiments revealed that anaerobic growth of PA14 $\Delta$ paiI was only retarded after $96 \mathrm{~h}$ when glucose was provided as the sole carbon source (data not shown). To study this further, we determined the CFU of PA14 and PA14 1 paiI during growth in BSM medium supplemented with glucose and $\mathrm{KNO}_{3}$ at several times after shift to anaerobiosis. As shown in Figure 4A, PA14 $\Delta$ paiI showed a reduced viability under anoxic conditions when compared to PA14. Ectopic expression of paiI in strain PA14 $\Delta$ paiI(pME-paiI) rescued the phenotype and restored the CFU comparable to that of PA14 (Figure 4B).

\section{Uptake of Glucose in PA14 $\Delta$ pail}

Since PA14 $\Delta$ paiI showed reduced viability in glucose minimal medium, we next asked whether the observed phenotype can be attributed to a decreased glucose uptake. To test this, the 

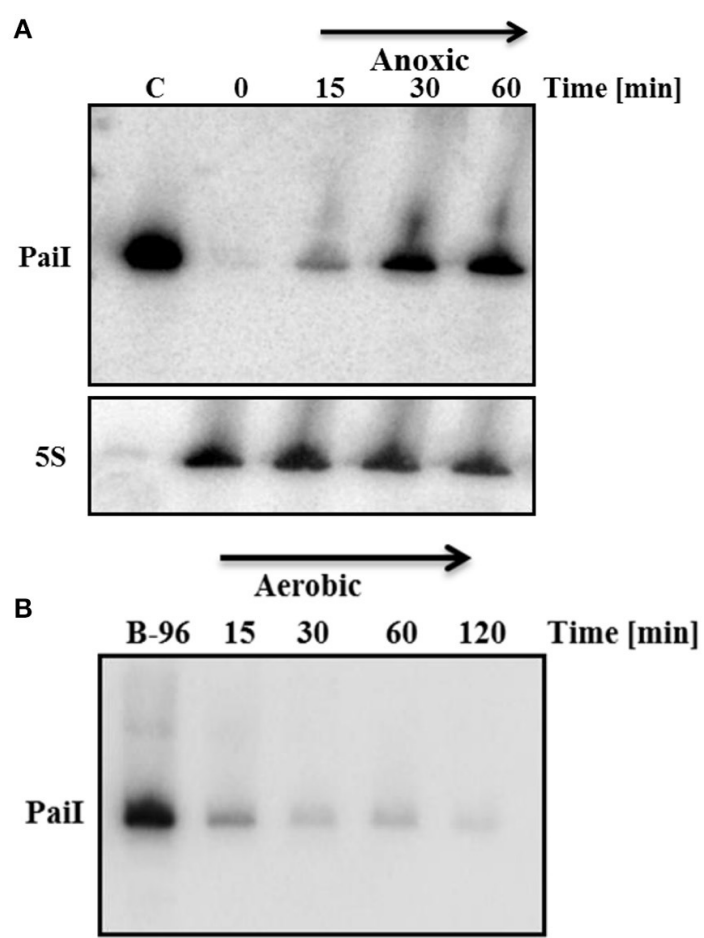

$5 \mathrm{~S}$

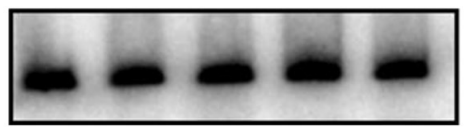

C

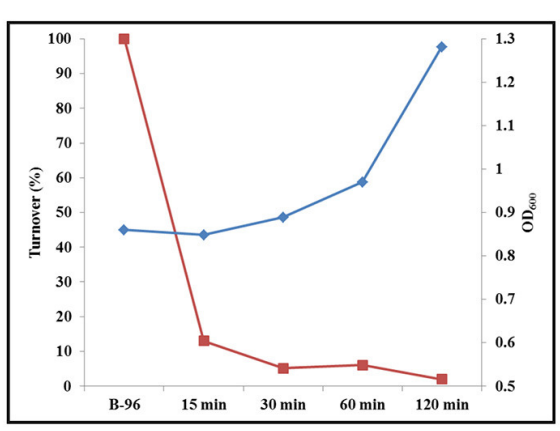

FIGURE 3 | Synthesis and turnover of Pail. (A) PA14 cultures were grown planktonically to an $\mathrm{OD}_{600}$ of 0.45 (0 min). Then, $\mathrm{KNO}_{3}$ was added to a final concentration of $100 \mathrm{mM}$ and the cultures were shifted to anoxic conditions. Total RNA was extracted at the indicated times followed by the detection of Pail by Northern-blotting. $0.6 \mathrm{ng}$ of in vitro transcribed Pail (126 nt) was loaded as a positive control (C). (B) PA14 B-96 cultures were shifted to aerobic conditions. Total RNA was extracted at the indicated times followed by the detection of Pail by Northern-blotting. (C) Graphical presentation of PA14 growth (blue graph) and Pail decay (red graph) based on the results shown in (B)

amount of glucose present in the supernatants of PA14 and PA14 $\Delta$ paiI was determined before $(0 \mathrm{~h})$ and 1 and $2 \mathrm{~h}$ after shift to anaerobiosis. As shown in Figure 5, the amount of glucose present in the supernatant of PA14 $\Delta$ paiI was somewhat higher than that observed for PA14. These results implied that the sRNA might impact on glucose uptake under anaerobic conditions.
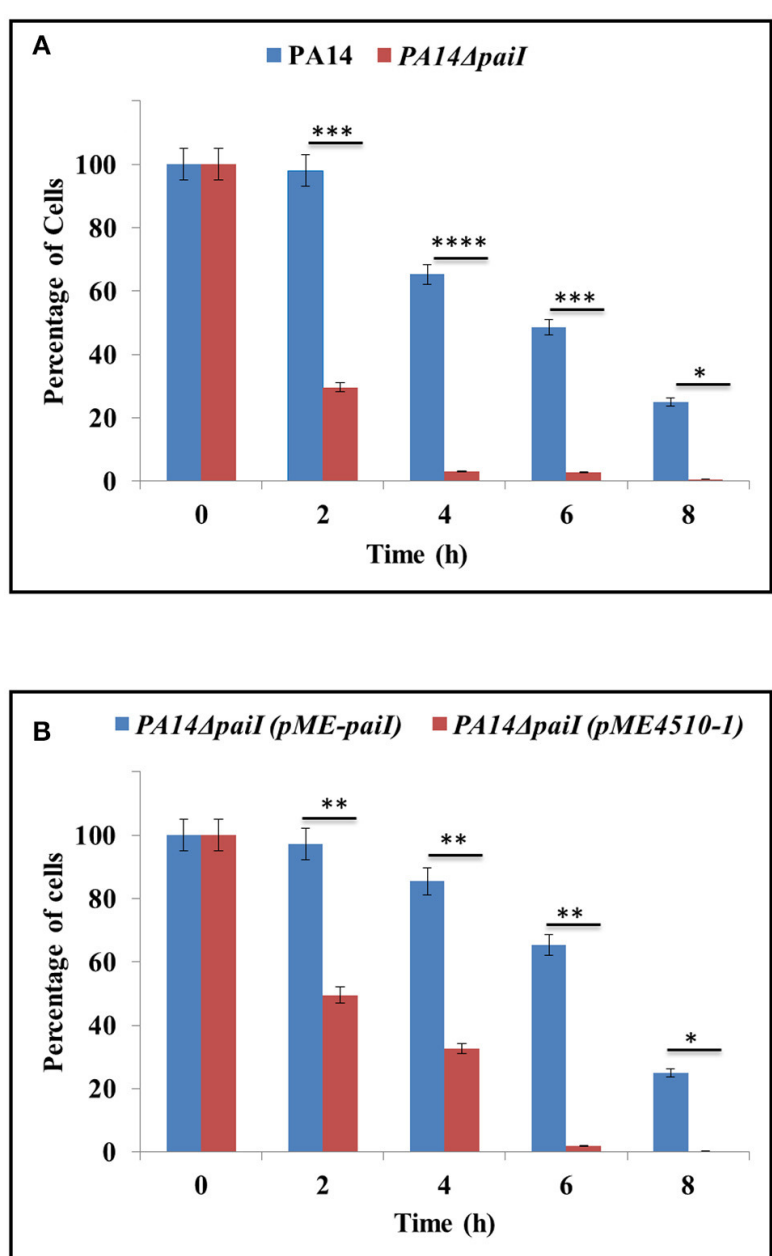

FIGURE 4 | Anaerobic growth of PA14 $\Delta$ pail. The strains were grown in BSM medium supplemented with $20 \mathrm{mM}$ glucose. The CFU at time 0 (shift from aerobic to anaerobic conditions and concomitant addition of $\mathrm{KNO}_{3}$ to a final concentration of $100 \mathrm{mM}$ ) was set to $100 \%$. The bars represent the percentage in CFU at either time relative to time 0 . The CFU were obtained by plating serial dilutions of the cultures at the indicated times on LB plates. (A) Decline in the CFU of PA14 and PA14 $\Delta$ pail after shift to anaerobiosis. (B) Decline in the CFU of PA14 $\Delta$ pail (pME-pail) and PA14 (pME4510-1) after shift to anaerobiosis. At time 0, IPTG was added to a final concentration of $2 \mathrm{mM}$ to both cultures to induce transcription of the plasmid borne pail gene in strain $\mathrm{PA} 14 \Delta$ pail(pME-pail). Depicted is mean $\pm \mathrm{SD}, n=3$. Statistical analysis was performed using the unpaired student $t$-test (www.graphpad.com). ${ }^{\star \star \star \star \star} p<$ $0.0001,{ }^{\star * *} p<0.001,{ }^{* *} p<0.01,{ }^{*} p<0.05$.

\section{Reduced Nitrite Reductase Activity in PA14 $\Delta$ pail}

As expression of pail was nitrate dependent (Supplementary Figure S4), we tested whether Pail impacts on the denitrification pathway. First, we asked whether PaiI affects the first step in denitrification, i.e., the conversion of $\mathrm{NO}_{3}$ to $\mathrm{NO}_{2}$, which is performed by the cytoplasmic nitrate reductase (Supplementary Figure S1). However, no significant difference in the nitrate reductase activity was observed in strains PA14 and PA14 1 paiI after shift to anaerobiosis (Supplementary Figure S5). 


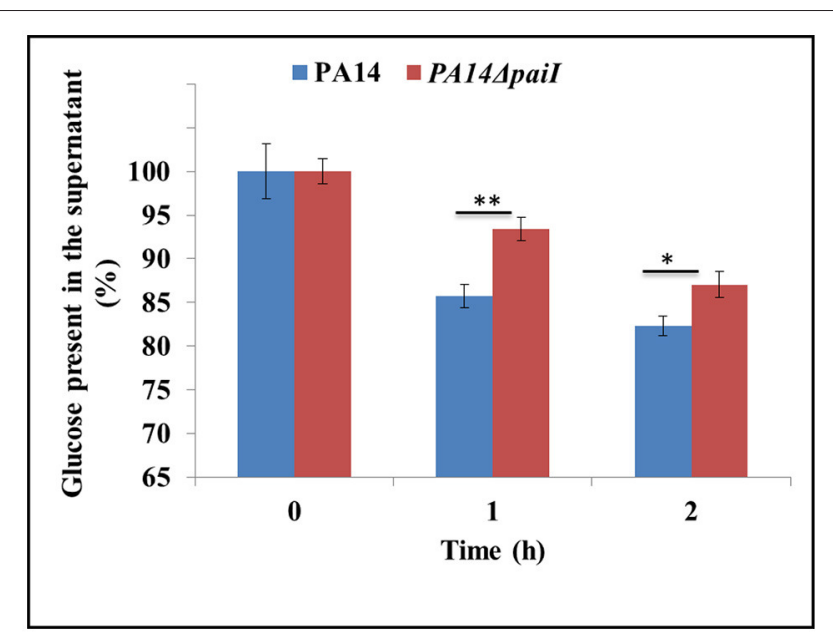

FIGURE 5 | Reduced glucose uptake by strain PA14 $\Delta$ pail. The amount of glucose present in the supernatant of PA14 and PA14 $\Delta$ pail cultures was determined under the same conditions as described in the legend to Figure 4. The glucose concentration at time 0 (shift from aerobic to anaerobic conditions and addition of $\mathrm{KNO}_{3}$ to a final concentration of $100 \mathrm{mM}$ ) was set to $100 \%$. The bars represent the percentage of glucose present at either time relative to time 0.). Depicted is mean $\pm \mathrm{SD}, n=9$. Statistical analysis was performed using the unpaired student $t$-test (www.graphpad.com). ${ }^{* \star} p<0.01,{ }^{*} p<0.05$.

Second, the $\mathrm{NO}_{2}$ accumulation was compared in the supernatants of PA14 and PA14 $\Delta$ paiI at different times after shift to anaerobiosis. Up to $3 \mathrm{~h}$ after shift to anaerobiosis, there was significantly more $\mathrm{NO}_{2}$ accumulation observed with PA14 $\Delta$ paiI than with the wild-type strain (Figure 6A). Ectopic expression of PaiI in strain PA14 $\Delta$ paiI(pME-paiI) complemented the paiI deletion phenotype (Figure 6B). As the difference in the $\mathrm{NO}_{2}$ levels observed with PA14 and PA14 $\Delta$ paiI were unlikely to result from a differential nitrate reductase activity (Supplementary Figure S5), we next tested whether PA14 $\Delta$ paiI displays differences in the activity of the nitrite reductase, which converts $\mathrm{NO}_{2}$ to $\mathrm{NO}$ (Supplementary Figure S1). These enzymatic assays revealed a reduced nitrite reductase activity in PA14 1 paiI when compared with PA14 during the first $3 \mathrm{~h}$ after shift to anaerobiosis (Figure 6C). Ectopic expression of paiI in strain PA14 1 paiI(pME-paiI) again complemented this phenotype (Figure 6D).

\section{Overexpression of $d n r$ Alleviates the pail Deletion Phenotype}

As the PA14 $\Delta$ paiI mutant showed a reduced nitrite reductase activity and higher levels of $\mathrm{NO}_{2}$ in the culture supernatant, we next asked whether increased synthesis of the transcriptional regulators ANR, DNR and NirQ can alleviate the paiI deletion phenotype. ANR positively regulates $d n r$, whereas DNR and NirQ are direct positive regulators of the nir operon, encoding the subunits of the nitrite reductase (Supplementary Figure S1; Schreiber et al., 2007). The corresponding genes were mounted into the expression vector pMMB67HE under transcriptional control of the tac promoter. The nitrite accumulation and nitrite reductase activity were then compared

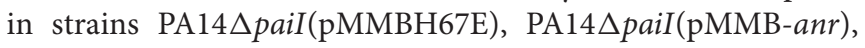

PA14 $\Delta$ paiI(pMMB-dnr) and PA14 $\Delta$ paiI(pMMB-nirQ). As shown in Figures 7A,B, over-expression of $d n r$ restored the nitrite reductase activity in PA14 $\Delta p a i I(\mathrm{pMMB}-d n r$ ) to wild-type levels, which was accompanied by a reduced $\mathrm{NO}_{2}$ accumulation. A similar effect was observed after anr over-expression albeit to a lower extend. In contrast, over-expression of nirQ did not impact on the $\mathrm{NO}_{2}$ accumulation and nitrite reductase activity (Figures 7A,B).

\section{Comparative Transcriptome Analysis of PA14 vs. PA14 $\Delta$ pail}

In order to identify target gene(s) for PaiI, an $\mathrm{RNA}_{\text {seq }}$ based transcriptome analysis was performed with total RNA isolated from PA14 and PA14 1 paiI after anaerobic growth for $1 \mathrm{~h}$ in BSM medium supplemented with glucose and $\mathrm{KNO}_{3}$. Only 19 transcripts (15 up- and 4 down-regulated) showed a $\geq \pm$ 1.5-fold change in PA14 when compared with PA14 14 paiI (Supplementary Table S4). However, none of the encoded genes are directly involved in the denitrification pathway. The only transcripts that might indirectly affect the denitrification pathway are those involved in sulfur metabolism (cys $A, c y s I$, and $c y s N)$, as iron sulfur clusters are required for the function of ANR and of the nitrate reductase activity (Zumft, 1997; Yoon et al., 2007). However, as the nitrate reductase activity was not impaired in the PA14 $\Delta$ paiI strain (Supplementary Figure S5), we did not follow up the idea that a scarcity of sulfur is responsible for the $\Delta p a i I$ phenotype. In addition, two chaperone genes ( $i b p A$ and groES) were significantly more abundant in PA14 when compared to PA14 $\Delta$ pail. IbpA is a heat shock protein, which is induced in response to several stress conditions like oxygen limitation in Bacillus subtilis (Hecker et al., 1996), toluene and other stresses in Pseudomonas putida (Matuszewska et al., 2008). We next investigated whether IbpA might impact on denitrification by comparing the anaerobic growth of PA14 with that of a PA14 $\triangle i b p A$ mutant strain in BSM medium supplemented with glucose. Growth was not impaired in the deletion strain (not shown), which apparently eliminates the possibility that this chaperone is involved in denitrification. Moreover, we observed that the mexG gene, which appears to be indirectly regulated by DNR (Trunk et al., 2010) was also down-regulated in PA14 $\Delta$ paiI. In addition, the $r s m A$ gene, encoding the translational regulator RsmA that is involved in regulation of virulence genes (Brencic and Lory, 2009) was up-regulated. Of the 4 transcripts that were down-regulated in the presence of Pail, two encode hypothetical proteins (PA14_11670 and PA14_13960). The other two genes encode a putative transposase (PA14_13970) and aquaporin Z porin $(a q p Z)$, respectively. Aquaporin $Z$ is selectively permeable to water and plays a role in osmoregulation (Calamita, 2000), however, it has so far not been linked to the denitrification pathway. In contrast to $E$. coli FnrS, which was reported to repress functions that are not required during anaerobiosis (Durand and Storz, 2010), we did not observe a significant down-regulation of such functions.

\section{PA14 1 pail Growth Is Impaired in Murine Tumors}

The above findings suggested that PaiI positively impacts on the denitrification pathway. This prompted us to test whether 

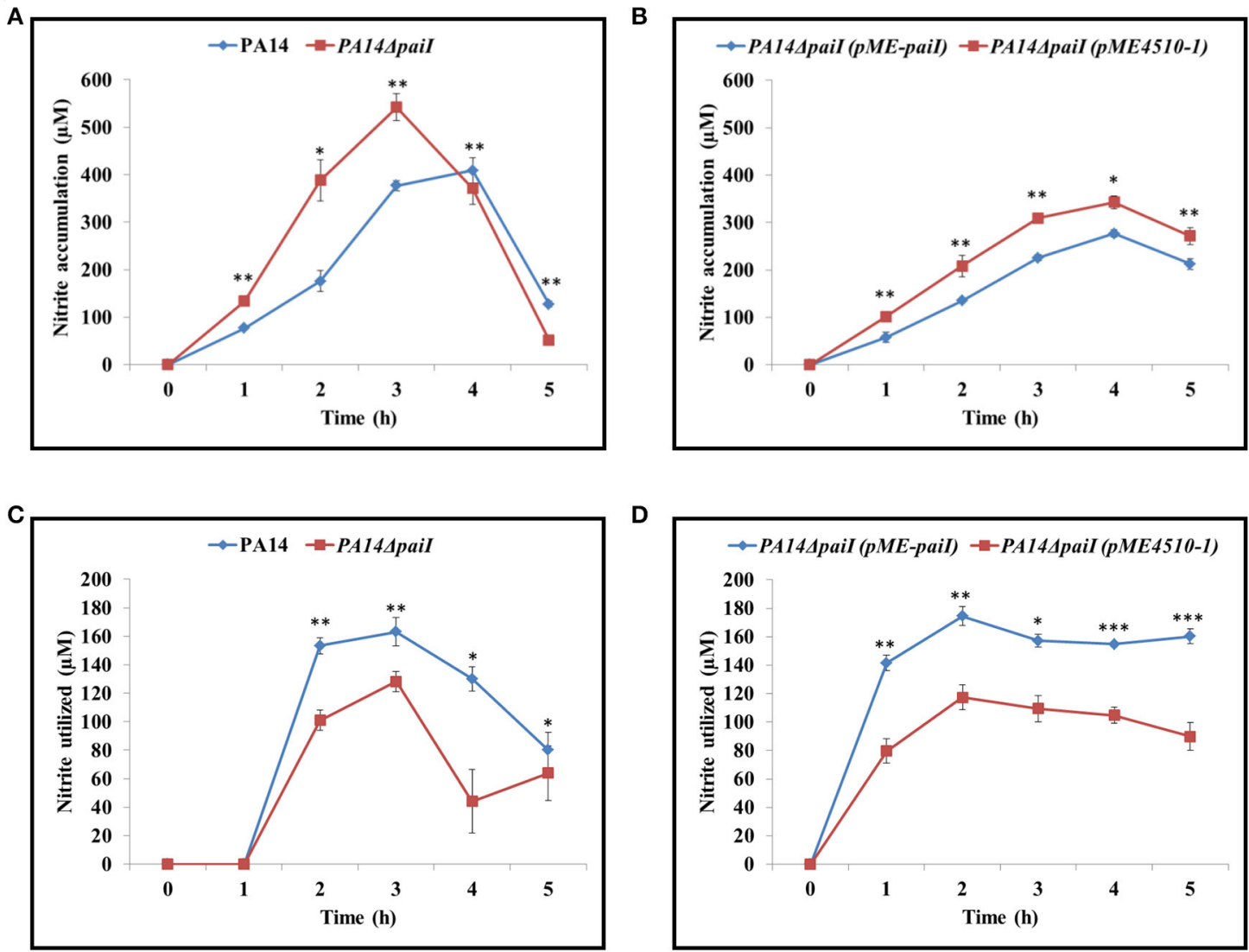

D

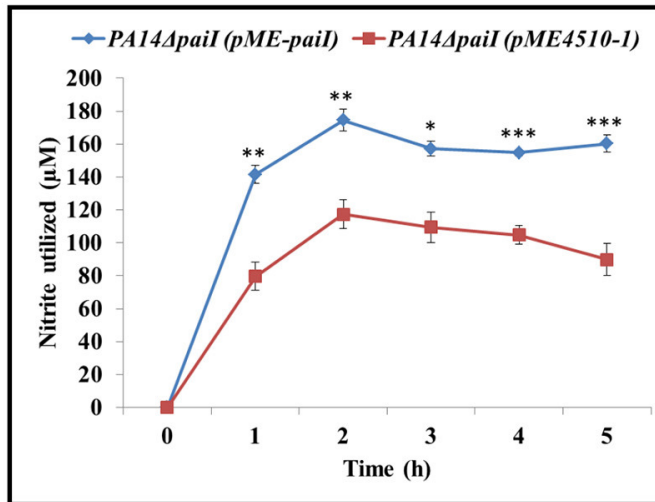

E

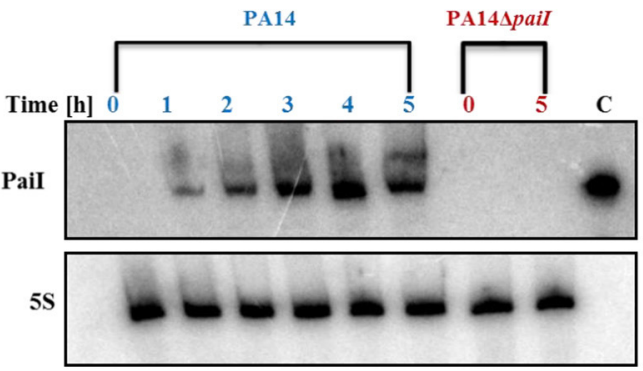

$\mathbf{F}$

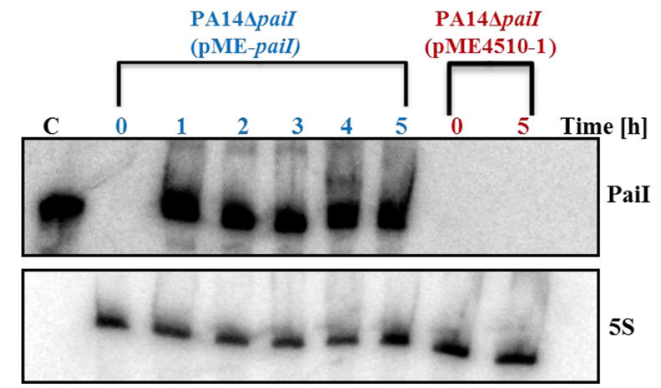

FIGURE 6 | $\mathrm{NO}_{2}$ accumulation and nitrite reductase activity obtained with strains PA14, PA14 $\Delta$ pail, and PA14 $\Delta$ pail(pME-pail). To determine the $\mathrm{NO}_{2}$ concentration in the supernatants $(\mathbf{A}, \mathbf{B})$ and the $\mathrm{NO}_{2}$ reductase activity $(\mathbf{C}, \mathbf{D})$, the cultures were grown under the same conditions as described in the legend to Figure 4. Depicted is mean $\pm \mathrm{SD}, n=3$. Statistical analysis was performed using the unpaired student $t$-test (www.graphpad.com). ${ }^{\star \star *} p<0.001,{ }^{* *} p<0.01,{ }^{*} p<0.05$. (E,F) Pail levels detected by Northern-blot analyses in total RNA isolated from the samples collected at the indicated times. For strains PA14 $\Delta$ pail and PA14 $\Delta$ pail (pME4510-1) only total RNA isolated at time 0 and $5 \mathrm{~h}$ were loaded onto the gel. $5 \mathrm{~S}$ RNA levels determined by Northern-blotting served as a loading control.

the paiI deletion strain is impaired in growth in CT26 murine tumors. In this model the capability to survive in hypoxic/anaerobic conditions within the tumor is crucial for tumor colonization (Komor et al., 2012). The BALB/c mice bearing an ectopic CT26 tumor were intravenously (i.v) infected with either PA14 or PA14 1 pail. At $24 \mathrm{~h}$ post-infection (p.i), bacterial colonization of the tumor was assessed by determination of the CFU. As shown in Figure 8, the pail deletion strain was impaired in colonizing the tumor. Thus, this experiment again underlined the function of PaiI in adaptation to anaerobic denitrification conditions.

\section{DISCUSSION}

Here, we report the first study on a Pseudomonas sRNA that is induced during nitrate respiration in a $\mathrm{NO}_{3}-/ \mathrm{NarXL}$ dependent manner (Figure 2, Supplementary Figure S4). In strain PA14, the paiI gene is situated in the intergenic region between PA14_13970 and PA14_13990 (Supplementary Figure S2A), which encodes a putative transposase and a component of a putative ABC transporter, respectively (Zhang et al., 2000). While the synteny of paiI and PA14_13990 is highly conserved in all sequenced 138 P. aeruginosa strains (Zhang et al., 2000), 

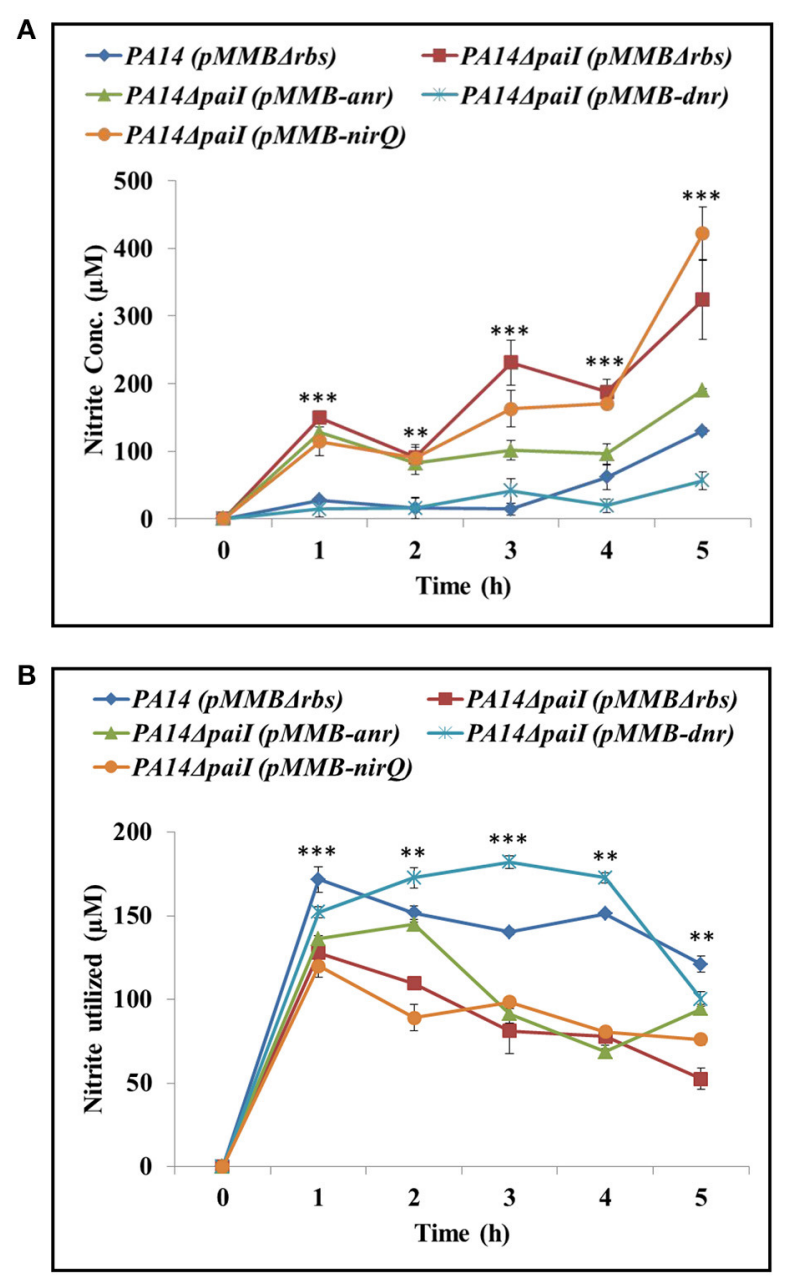

FIGURE 7 | Ectopic expression of anr and $d n r$ complement the $\Delta$ pail phenotype. (A), $\mathrm{NO}_{2}$ levels in the supernatants and (B) nitrite reductase activity determined with strains PA14 $\Delta$ pail(pMMB-anr), PA14 $\Delta$ pail(pMMB-dnr) and PA14 $\Delta$ pail(pMMB-nirQ). PA14 and PA14 $\Delta$ pail strains carrying the empty vector $\mathrm{pMMB} \Delta r b s$ were used as a control. The cultures were grown under the same conditions as described in the legend to Figure 4. Depicted is mean \pm $\mathrm{SD}, n=3$. Statistical analysis was performed using ordinary one-way ANOVA (www.graphpad.com). ${ }^{* \star *} p<0.001,{ }^{* *} p<0.01$.

the putative transposon gene is only present in 24 (Zhang et al., 2000). Pail was detectable after shift to anaerobiosis (Figure 3A) and was rapidly degraded after shift to aerobic conditions (Figure 3B). Unfortunately, the use of mutant strains of the Liberati transposon library (Liberati et al., 2006) with insertions in the genes encoding the enzymes RNase E, CafA, RNase III, RNase R, and RNase HII did not allow to pin down a particular RNase involved in degradation of the sRNA, as it was rapidly degraded upon aerobic shift in either mutant (not shown).

A detectable phenotype of the paiI deletion strain was only observed during anaerobiosis when glucose was provided as the sole carbon source (Figure 4), which can be reconciled with a somewhat reduced uptake of glucose (Figure 5). However, the transcriptome analysis did not reveal significant differences in the

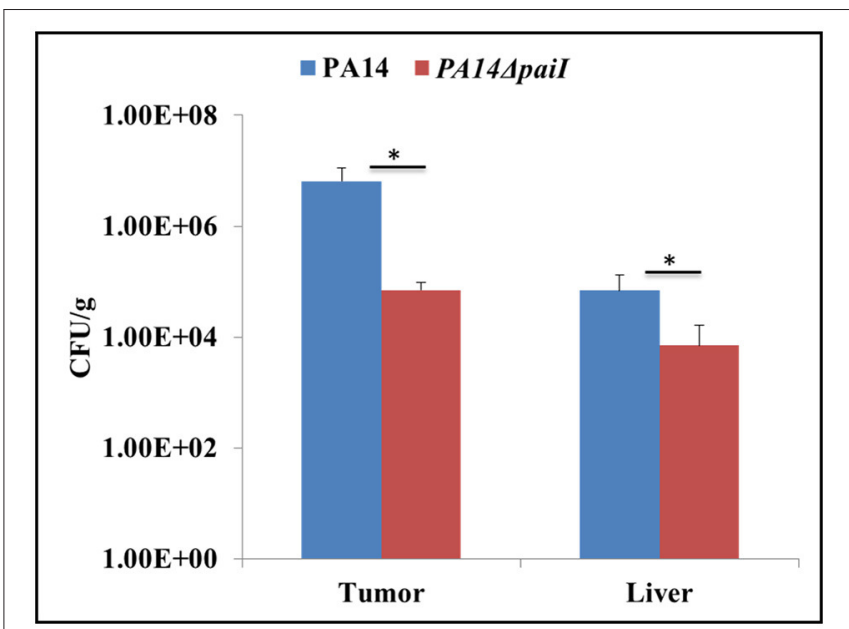

FIGURE 8 | Bacterial colonization of CT26 tumors. Tumor-bearing mice were infected intravenously with $P$. aeruginosa PA14 and PA14 $\Delta$ pail. At 24 h the tumor and liver were homogenized and the CFU per gram of tissue were determined. Depicted is mean $\pm \mathrm{SD}, n=5$. Statistical analysis was performed using the unpaired student $t$-test (www.graphpad.com). ${ }^{*} p<0.05$.

transcript levels of the major functions involved in glucose uptake such as oprB, gltB,F,G,K in PA14 and PA14 $\Delta$ paiI (Supplementary Table S4), which indicated that Pail does not directly impact on the expression of these genes. In the absence of Pail, an increased transient accumulation of nitrite was observed in the medium, which concurred with a reduced activity of the nitrite reductase (Figure 6). It has been reported that glucose uptake under denitrifying conditions corresponds to the maximal levels of nitrate and nitrite reductase activities, and coincided with the disappearance of nitrite from the medium (Williams et al., 1978). The latter authors interpreted this as showing that glucose uptake is directly linked to the activity of these enzymes, i.e., to the levels of nitrate and nitrite. As the activity of the nitrate reductase was not impaired in PA14 $\Delta$ paiI (Supplementary Figure S5), it seems likely that the lowered uptake of glucose resulted from the reduced activity of the nitrite reductase, i.e., from increased levels of nitrite. However, the molecular details underlying nitritelinked glucose transport remain unknown in Pae.

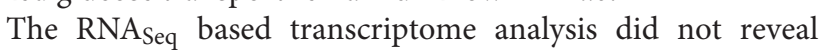
significant differences in the levels of the nir transcripts in PA14 1 paiI when compared with the wild-type strain (Supplementary Table S4). However, the transcript levels may not always mirror the translational output of a given gene. Among other regulatory events, sRNAs can positively regulate translation by interfering with intramolecular secondary structures that obstructs the translational initiation signals, i.e. the Shine and Dalgarno (SD) sequence or the start codon (Reichenbach et al., 2008; Sonnleitner et al., 2011). As indicated in Supplementary Figure S6A, PaiI displays sequence complementarity to a region upstream of the $\mathrm{SD}$ sequence of nirS mRNA that might form a stem-loop structure, which could potentially impact on its translational efficiency. To test whether PaiI positively affects translation of nirS, the nitrite reductase levels were assessed by quantitative western-blotting 
with anti-NirS antibodies. No apparent difference in the NirS levels were observed in strain PA14 when compared with strain PA14 $\Delta$ paiI (Supplementary Figure S6B), excluding the possibility that PaiI directly regulates the translational output of nirS mRNA.

The increased nitrite levels in the medium and the reduced nitrite reductase activity observed with the PA14 $\Delta$ pail strain were alleviated by ectopic expression of the $a n r$ and $d n r$ genes (Figure 7). Hence, the observed restoration of the nitrite reductase activity could be simply based on a gene dosage effect, i.e., an increased transcription of nirS mRNA through overproduction of DNR (see Supplementary Figure S1) followed by a concomitant increase of the NirS levels. However, we did not observe an increased production of NirS in strain PA14 $\Delta$ paiI harboring plasmid pMMB-dnr (Supplementary Figure S6B). Interactome studies revealed that a number of proteins involved in several metabolic pathways associate together with denitrification enzymes to form a nitrate respirasome (BorreroDe Acuña et al., 2016). In addition, there are a number of accessory proteins that have been implicated in denitrification by genetic analyses (Zumft, 1997) and fitness studies (Vaccaro et al., 2015). This raises the possibility that both, DNR and Pail, regulate a hitherto unknown accessory factor that affects the function of nitrite reductase. Ectopic expression of $a n r$, encoding the anerobic regulator ANR, resulted also in partial complementation of the $\Delta$ paiI phenotype (Figure 7). The simplest explanation is the positive control of DNR by ANR (see Supplementary Figure S1; Schreiber et al., 2007) as all genes that are DNR dependent are also dependent on ANR (Trunk et al., 2010). In contrast, ectopic expression of nirQ did not restore the $\Delta$ paiI phenotype albeit it was shown to positively control nirS transcription (Hayashi et al., 1998). We can only speculate that NirQ does not impact on production of the hypothetical accessory factor for NirS function.

There are other possibilities how PaiI might indirectly affect the activity of nitrite reductase. One possibility for the reduced nitrite reductase activity observed in PA14 $\Delta$ paiI are the increased nitrite levels, which might negatively impact on the heme moiety of the enzyme (Rowe et al., 1977). Because of its toxicity nitrite is excreted to the periplasm, where the nitrite reductase converts it to nitric oxide (Schreiber et al., 2007). Thus, the compartmentalization of nitrite might explain why the activity of the nitrite reductase was affected in the PA14 $\Delta$ paiI but not that of the inner membrane bound nitrate reductase, the NarI subunit of which also contains a heme group (Van Alst et al., 2009). Moreover, Yoon et al. (2002) have reported that a Pae O1oprF mutant lacked nitrite reductase activity, whereas the underlying mechanism remains speculative. However, the transcriptome data did not reveal significant changes in the transcript levels of $\operatorname{oprF}$ and no apparent differences in the OprF levels were observed by quantitative Western-blotting (not shown) in the absence or presence of Pail, respectively. Thus, these data would argue against the possibility that PaiI acts on the nitrate reductase activity through regulation of oprF.

Although under laboratory conditions the $\Delta p a i I$ phenotype was only obvious when glucose was used as the sole carbon source, it was striking that the absence of Pail resulted in a fitness burden in the mouse tumor model (Figure 8). A PA14 anr mutant was found to colonize the tumor at lower bacterial numbers and did not colonize the hypoxic/anaerobic central necrotic areas of the tumor which emphasized the importance to adapt to microaerophilic/anaerobic growth conditions for efficient tumor colonization (Komor et al., 2012). Comparative transcriptional profiling of Pae indicated physiological similarity of the bacteria in the murine tumor model and in the cystic fibrosis (CF) lung (Bielecki et al., 2013). Thus, it appears possible that Pail also contributes to the adaptation of Pae to conditions present in the CF lung, where Pae can grow anaerobically (Kolpen et al., 2014).

\section{AUTHOR CONTRIBUTIONS}

Conceived and designed the experiments: MT and UB. Performed the experiments: MT and VP. Analyzed the data: MT, MW, FA, SH, SW, and UB. Contributed reagents/materials/analysis tools: $\mathrm{SW}, \mathrm{SH}$, and $\mathrm{UB}$. Wrote the paper: MT and UB.

\section{FUNDING}

The work was supported by the Austrian Science Fund (www.fwf. ac.at/en) through the Special Research Program RNA-REG F43, subproject AF4311(UB), and the doctoral program RNA-Biology W-1207 (MT).

\section{ACKNOWLEDGMENTS}

We are grateful to Mrs. S. Buchmaier and Dr. G. Layer for providing the anti-NirS antibodies.

\section{SUPPLEMENTARY MATERIAL}

The Supplementary Material for this article can be found online at: https://www.frontiersin.org/articles/10.3389/fmicb. 2017.02312/full\#supplementary-material

Supplementary Figure S1 | Anaerobic respiration in P. aeruginosa. ANR acts as the master oxygen-sensing regulator, which activates transcription of the nar $X L$ operon, encoding a two-component nitrate responsive two-component system. Under low-oxygen tension and in the presence of nitrate, ANR and NarL activate the transcription of the narK1K2GHJl operon encoding nitrate/nitrite transporters, and the structural genes for the respiratory nitrate reductase, which converts nitrate into nitrite. ANR and NarL co-operatively activate the $d n r$ gene encoding the $\mathrm{NO}_{2} / \mathrm{NO}$ responsive regulator DNR. DNR activates the transcription of all other denitrification genes, encoding enzyme complexes that catalyze the conversion of nitrate into $\mathrm{N}_{2}$. NirQ requires DNR and NarL for its synthesis, and is predicted to be involved in fine tuning of the nitrite reductase activities. Nar, Nir, Nor, and Nos indicate nitrate reductase, nitrite reductase, nitric oxide reductase, and nitrous oxide reductase, respectively (adapted from Schreiber et al., 2007).

Supplementary Figure S2 | (A) Genomic location of pail and read coverage of the pail transcript under conditions P, A-30 and B-96 visualized by the Genome Browser. (B) The transcriptional start site (TSS) of the pail gene was determined by primer extension analysis with total RNA isolated from B-96 cells of PA14 (Lane 1). Sequence reactions (T, G, $C$, and $A$ ) were performed with the same primer using in vitro transcribed Pail-1 (141 nt) sRNA as template and run in parallel. The transcriptional start $(+1 \mathrm{~A})$ is indicated on the left. The upstream and downstream sequences are shown on the right. F, primer extension signal obtained for the in 
vitro transcribed Pail-1 RNA (141 nt). (C) Genetic locus encoding Pail. The-10 and-35 promoter regions are colored in green, the transcriptional start site (TSS) and the NarL binding sites are show in red and violet, respectively. The consensus NarL signature (Schreiber et al., 2007) is shown boxed. The deleted NarL binding site within the pail promoter sequence in strain PA14 $\Delta$ narL-pail is in violet. The numbers refer to the PA14 genome coordinates (http://www.pseudomonas.com).

Supplementary Figure S3 | Pail Secondary structure. (A) Predicted Pail secondary structure according to RNAfold WebServer (http://rna.tbi.univie.ac.at/ cgi-bin/RNAWebSuite/RNAfold.cgi). (B) Mapping of T1 cleavage sites in Pail. Lane 1, alkaline ladder. Lanes 2, 3, and 4, $\mathrm{P}^{32}$ labeled Pail was treated with $0.2 \mathrm{U}$ of RNase T1 for 10 min, 15 min and 0 min respectively. Arrows depict the RNase T1 cleavage sites. Right, secondary structure of Pail derived from the assay.

Supplementary Figure S4 | Nitrate dependent synthesis of Pail. Detection of Pail by Northern-blotting in total RNA extracted from strain PA14 after $96 \mathrm{~h}$ of anoxic growth in BSM medium supplemented with $20 \mathrm{mM}$ glucose. (+) and (-), presence and absence of nitrate in the medium, respectively.
Supplementary Figure S5 | Nitrate reductase activity of strains PA14 and PA14 $\triangle$ pail. The nitrate reductase activity was determined in whole cell extracts of PA14 and PA14 $\Delta$ pail. The cultures were grown under the same conditions as described in the legend to Figure 4.

Supplementary Figure S6 | (A) Putative base-pairing between Pail and nirS mRNA. Putative secondary structure of the nirS mRNA upstream of the start codon predicted by Mfold (http://unafold.rna.albany.edu/?q=mfold/RNA-FoldingForm2.3). The AUG start codon is shown boxed and the Shine and Dalgarno sequence (SD) is indicated by a red bar. The putative Pail interacting region is indicated by a blue bar. (B) Pail and DNR-overproduction does not affect the NirS levels. The strains PA14, PA14 $\Delta$ pail, PA14 $\Delta$ pail(pMMB $\Delta r b s)$, and $\mathrm{PA} 14 \Delta$ pail(pMMB-dnr) were grown as described in the legend to Figure 4. At the indicated times after shift to anaerobiosis, samples were withdrawn and equal amounts of protein were separated on SDS-polyacrylamide gels. NirS was detected with anti-NirS antibodies. Ribosomal protein S2 served as a loading control. Lane M, molecular weight markers.

\section{REFERENCES}

Ausubel, F., Brent, R., Kingston, R. E., Moore, D. D., Seidman, J. G., Smith, J. A., et al. (1997). Short Protocols in Molecular Biology, 3rd Edn. New York, NY: John Wiley \& Sons, Inc.

Bielecki, P., Komor, U., Bielecka, A., Müsken, M., Puchalka, J., Pletz, M. W., et al. (2013). Ex vivo transcriptional profiling reveals a common set of genes important for the adaptation of Pseudomonas aeruginosa to chronically infected host sites. Environ. Microbiol. 15, 570-587. doi: 10.1111/1462-2920.12024

Bolger, A. M., Lohse, M., and Usadel, B. (2014). Trimmomatic: a flexible trimmer for Illumina sequence data. Bioinformatics 30, 2114-2120. doi: 10.1093/bioinformatics/btu170

Borrero-De Acuña, J. M., Rohde, M., Wissing, J., Jänsch, L., Schobert, M., Molinari, G., et al. (2016). Protein network of the Pseudomonas aeruginosa denitrification apparatus. J. Bacteriol. 198, 1401-1413. doi: 10.1128/JB.00055-16

Boysen, A., Møller-Jensen, J., Kallipolitis, B., Valentin-Hansen, P., and Overgaard, M. (2010). Translational regulation of gene expression by an anaerobically induced small non-coding RNA in Escherichia coli. J. Biol. Chem. 285, 10690-10702. doi: 10.1074/jbc.M109.089755

Brencic, A., and Lory, S. (2009). Determination of the regulon and identification of novel mRNA targets of Pseudomonas aeruginosa RsmA. Mol. Microbiol. 72, 612-632. doi: 10.1111/j.1365-2958.2009.06670.x

Calamita, G. (2000). The Escherichia coli aquaporin-Z water channel. Mol. Microbiol. 37, 254-262. doi: 10.1046/j.1365-2958.2000.02016.x

Caldelari, I., Chao, Y., Romby, P., and Vogel, J. (2013). RNA-mediated regulation in pathogenic bacteria. Cold Spring Harb. Perspect. Med. 3:a010298. doi: 10.1101/cshperspect.a010298

Durand, S., and Storz, G. (2010). Reprogramming of anaerobic metabolism by the FnrS small RNA. Mol. Microbiol. 75, 1215-1231. doi: 10.1111/j.1365-2958.2010.07044.x

Durham, D. R., and Phibbs, P. V. Jr. (1982). Fractionation and characterization of the phosphoenolpyruvate: fructose 1-phosphotransferase system from Pseudomonas aeruginosa. J. Bacteriol. 149, 534-541.

Farnham, P. J., and Platt, T. (1981). Rho-independent termination: dyad symmetry in DNA causes RNA polymerase to pause during transcription in vitro. Nucleic Acids Res. 9, 563-577. doi: 10.1093/nar/9.3.563

Ferrara, S., Brugnoli, M., De Bonis, A., Righetti, F., Delvillani, F., Dehò, G., et al. (2012). Comparative profiling of Pseudomonas aeruginosa strains reveals differential expression of novel unique and conserved small RNAs. PLoS ONE 7:e36553. doi: 10.1371/journal.pone.0036553

Ferrara, S., Carloni, S., Fulco, R., Falcone, M., Macchi, R., and Bertoni, G. (2015). Post-transcriptional regulation of the virulence-associated enzyme AlgC by the sigma(22) -dependent small RNA ErsA of Pseudomonas aeruginosa. Environ. Microbiol. 17, 199-214. doi: 10.1111/1462-2920.12590

Figurski, D. H., and Helinski, D. R. (1979). Replication of an origin-containing derivative of plasmid RK2 dependent on a plasmid function provided in trans. Proc. Natl. Acad. Sci. U.S.A. 76, 1648-1652. doi: 10.1073/pnas.76.4.1648

Franch, T., Thisted, T., and Gerdes, K. (1999). Ribonuclease III processing of coaxially stacked RNA helices. J. Biol. Chem. 274, 26572-26578. doi: $10.1074 /$ jbc.274.37.26572

Fürste, J. P., Pansegrau, W., Frank, R., Blöcker, H., Scholz, P., Bagdasarian, M., et al. (1986). Molecular cloning of the plasmid RP4 primase region in a multi-host-range tacP expression vector. Gene 48, 119-131. doi: 10.1016/0378-1119(86)90358-6

Gottesman, S., and Storz, G. (2011). Bacterial small RNA regulators: versatile roles and rapidly evolving variations. Cold Spring Harb. Perspect. Biol. 3:a003798. doi: 10.1101/cshperspect.a003798

Hassett, D. J., Sutton, M. D., Schurr, M. J., Herr, A. B., Caldwell, C. C., and Matu, J. O. (2009). Pseudomonas aeruginosa hypoxic or anaerobic biofilm infections within cystic fibrosis airways. Trends Microbiol. 17, 130-138. doi: 10.1016/j.tim.2008.12.003

Hayashi, N. R., Arai, H., Kodama, T., and Igarashi, Y. (1998). The nirQ gene, which is required for denitrification of Pseudomonas aeruginosa, can activate the RubisCO from Pseudomonas hydrogenothermophila. Biochim. Biophys. Acta 1381, 347-350. doi: 10.1016/S0304-4165(98)00045-2

Hecker, M., Schumann, W., and Völker, U. (1996). Heat-shock and general stress response in Bacillus subtilis. Mol. Microbiol. 19, 417-428. doi: 10.1046/j.1365-2958.1996.396932.x

Hoe, C. H., Raabe, C. A., Rozhdestvensky, T. S., and Tang, T. H. (2013). Bacterial sRNAs: regulation in stress. Int. J. Med. Microbiol. 303, 217-229. doi: 10.1016/j.ijmm.2013.04.002

Hoffmann, S., Otto, C., Doose, G., Tanzer, A., Langenberger, D., Christ, S., et al. (2014). A multi-split mapping algorithm for circular RNA, splicing, trans-splicing and fusion detection. Genome Biol. 15:R34. doi: 10.1186/gb-2014-15-2-r34

Hoffmann, S., Otto, C., Kurtz, S., Sharma, C. M., Khaitovich, P., Vogel, J., et al. (2009). Fast mapping of short sequences with mismatches, insertions and deletions using index structures. PLoS Comput. Biol. 5:e1000502. doi: 10.1371/journal.pcbi.1000502

Holloway, B. W., Krishnapillai, V., and Morgan, A. F. (1979). Chromosomal genetics of Pseudomonas. Microbiol. Rev. 43, 73-102.

Kent, W. J., Sugnet, C. W., Furey, T. S., Roskin, K. M., Pringle, T. H., Zahler, A. M., et al. (2002). The human genome browser at UCSC. Genome Res. 12, 996-1006. doi: $10.1101 /$ gr.229102

Kolpen, M., Kühl, M., Bjarnsholt, T., Moser, C., Hansen, C. R., Liengaard, L., et al. (2014). Nitrous oxide production in sputum from cystic fibrosis patients with chronic Pseudomonas aeruginosa lung infection. PLoS ONE 9:e84353. doi: 10.1371/journal.pone.0084353

Komor, U., Bielecki, P., Loessner, H., Rohde, M., Wolf, K., Westphal, K., et al (2012). Biofilm formation by Pseudomonas aeruginosa in solid murine tumors - a novel model system. Microbes Infect. 14, 951-958. doi: 10.1016/j.micinf.2012.04.002

Lapouge, K., Schubert, M., Allain, F. H., and Haas, D. (2008). Gac/Rsm signal transduction pathway of gamma-proteobacteria: from RNA 
recognition to regulation of social behaviour. Mol. Microbiol. 67, 241-253. doi: 10.1111/j.1365-2958.2007.06042.x

Liberati, N. T., Urbach, J. M., Miyata, S., Lee, D. G., Drenkard, E., Wu, G., et al. (2006). An ordered, nonredundant library of Pseudomonas aeruginosa strain PA14 transposon insertion mutants. Proc. Natl. Acad. Sci. U.S.A. 103, 2833-2838. doi: 10.1073/pnas.0511100103

Livny, J., Brencic, A., Lory, S., and Waldor, M. K. (2006). Identification of 17 Pseudomonas aeruginosa sRNAs and prediction of sRNA-encoding genes in 10 diverse pathogens using the bioinformatic tool sRNAPredict2. Nucleic Acids Res. 34, 3484-3493. doi: 10.1093/nar/gkl453

Love, M. I., Huber, W., and Anders, S. (2014). Moderated estimation of fold change and dispersion for RNA-seq data with DESeq2. Genome Biol. 15:550. doi: 10.1186/s13059-014-0550-8

Matuszewska, E., Kwiatkowska, J., Kuczynska-Wisnik, D., and Laskowska, E. (2008). Escherichia coli heat-shock proteins IbpA/B are involved in resistance to oxidative stress induced by copper. Microbiology 154, 1739-1747. doi: 10.1099/mic.0.2007/014696-0

Miller, C. L., Romero, M., Karna, S. L., Chen, T., Heeb, S., and Leung, K. P. (2016). RsmW, Pseudomonas aeruginosa small non-coding RsmA-binding RNA upregulated in biofilm versus planktonic growth conditions. BMC Microbiol. 16, 155. doi: 10.1186/s12866-016-0771-y

Moir, J. W. B., Baratta, D., Richardson, D. J., and Ferguson, S. J. (1993). The purification of a cd1-type nitrite reductase from, and the absence of a coppertype nitrite reductase from, the aerobic denitrifier thiosphaera pantotropha; the role of pseudoazurin as an electron donor. Eur. J. Biochem. 212, 377-385. doi: 10.1111/j.1432-1033.1993.tb17672.x

Nicholas, D. J. D., Nason, A. (1957). "Determination of nitrate and nitrite," in Methods Enzymology, Vol. 3, eds S. P. Colowick and N. O. Kaplan (New York, NY: Academic Press), 1981-1984.

Nicke, T., Schnitzer, T., Münch, K., Adamczack, J., Haufschildt, K., Buchmeier, S., et al. (2013). Maturation of the cytochrome cd1 nitrite reductase NirS from Pseudomonas aeruginosa requires transient interactions between the three proteins NirS, NirN and NirF. Biosci. Rep. 33, 529-539. doi: 10.1042/BSR20130043

Palmer, K. L., Aye, L.M., and Whiteley, M. (2007). Nutritional cues control Pseudomonas aeruginosa multicellular behavior in cystic fibrosis sputum. J. Bacteriol. 189, 8079-8087. doi: 10.1128/JB.01 138-07

Pawar, V., Komor, U., Kasnitz, N., Bielecki, P., Pils, M. C., Gocht, B., et al. (2015). In vivo efficacy of antimicrobials against biofilm-producing Pseudomonas aeruginosa. Antimicrob. Agents Chemother. 59, 4974-4981. doi: 10.1128/AAC.00194-15

Pusic, P., Tata, M., Wolfinger, M. T., Sonnleitner, E., Häussler, S., and Bläsi, U. (2016). Cross-regulation by CrcZ RNA controls anoxic biofilm formation in Pseudomonas aeruginosa. Sci. Rep. 6:39621. doi: 10.1038/srep39621

Quinlan, A. R., and Hall, I. M. (2010). BEDTools: a flexible suite of utilities for comparing genomic features. Bioinformatics 26, 841-842. doi: 10.1093/bioinformatics/btq033

Reichenbach, B., Maes, A., Kalamorz, F., Hajnsdorf, E., and Görke, B. (2008). The small RNA GlmY acts upstream of the sRNA GlmZ in the activation of glmS expression and is subject to regulation by polyadenylation in Escherichia coli. Nucleic Acids Res. 36, 2570-2580. doi: 10.1093/nar/gkn091

Reinhart, A. A., Powell, D. A., Nguyen, A. T., O’neill, M., Djapgne, L., Wilks, A., et al. (2015). The prrF-encoded small regulatory RNAs are required for iron homeostasis and virulence of Pseudomonas aeruginosa. Infect. Immun. 83, 863-875. doi: 10.1128/IAI.02707-14

Rist, M., and Kertesz, M. A. (1998). Construction of improved plasmid vectors for promoter characterization in Pseudomonas aeruginosa and other gram-negative bacteria. FEMS Microbiol. Lett. 169, 179-183. doi: 10.1111/j.1574-6968.1998.tb13315.x

Rowe, J. J., Sherr, B. F., Payne, W. J., and Eagon, R. G. (1977). A unique nitric oxide-binding complex formed by denitrifying Pseudomonas aeruginosa. Biochem. Biophys. Res. Commun. 77, 253-258. doi: 10.1016/S0006-291X(77) 80190-3

Sambrook, J., and Russell, D. (2001). Molecular Cloning: A Laboratory Manual. New York, NY: Cold Spring Harbor Press.

Schreiber, K., Krieger, R., Benkert, B., Eschbach, M., Arai, H., Schobert, M., et al. (2007). The anaerobic regulatory network required for
Pseudomonas aeruginosa nitrate respiration. J. Bacteriol. 189, 4310-4314. doi: 10.1128/JB.00240-07

Sonnleitner, E., and Bläsi, U. (2014). Regulation of Hfq by the RNA CrcZ in Pseudomonas aeruginosa carbon catabolite repression. PLoS Genet. 10:e1004440. doi: 10.1371/journal.pgen.1004440

Sonnleitner, E., Gonzalez, N., Sorger-Domenigg, T., Heeb, S., Richter, A. S., Backofen, R., et al. (2011). The small RNA PhrS stimulates synthesis of the Pseudomonas aeruginosa quinolone signal. Mol. Microbiol. 80, 868-885. doi: 10.1111/j.1365-2958.2011.07620.x

Sonnleitner, E., and Haas, D. (2011). Small RNAs as regulators of primary and secondary metabolism in Pseudomonas species. Appl. Microbiol. Biotechnol. 91, 63-79. doi: 10.1007/s00253-011-3332-1

Stewart, V., and Parales, J. Jr. (1988). Identification and expression of genes narL and narX of the nar (nitrate reductase) locus in Escherichia coli K-12. J. Bacteriol. 170, 1589-1597. doi: 10.1128/jb.170.4.1589-1597.1988

Storz, G., Vogel, J., and Wassarman K. M. (2011). Regulation by small RNAs in bacteria: expanding frontiers. Mol. Cell 43, 880-891. doi: 10.1016/j.molcel.2011.08.022

Tata, M., Wolfinger, M. T., Amman, F., Roschanski, N., Dötsch, A., Sonnleitner, E., et al. (2016). RNASeq based transcriptional profiling of Pseudomonas aeruginosa PA14 after short- and long-term anoxic cultivation in synthetic cystic fibrosis sputum medium. PLoS ONE 11:e0147811. doi: 10.1371/journal.pone.0147811

Trunk, K., Benkert, B., Quäck, N., Münch, R., Scheer, M., Garbe, J., et al. (2010). Anaerobic adaptation in Pseudomonas aeruginosa: definition of the Anr and Dnr regulons. Environ. Microbiol. 12, 1719-1733. doi: 10.1111/j.1462-2920.2010.02252.x

Vaccaro, B. J., Thorgersen, M. P., Lancaster, W. A., Price, M. N., Wetmore, K. M., Poole, I. I., et al. (2015). Determining roles of accessory genes in denitrification by mutant fitness analyses. Appl. Environ. Microbiol. 82, 51-61. doi: 10.1128/AEM.02602-15

Van Alst, N. E., Sherrill, L. A., Iglewski, B. H., and Haidaris, C. G. (2009). Compensatory periplasmic nitrate reductase activity supports anaerobic growth of Pseudomonas aeruginosa PAO1 in the absence of membrane nitrate reductase. Can. J. Microbiol. 55, 1133-1144. doi: 10.1139/ W09-065

Vogel, J., and Luisi, B. F. (2011). Hfq and its constellation of RNA. Nat. Rev. Microbiol. 9, 578-589. doi: 10.1038/nrmicro2615

Voisard, C., Bull, C. T., Keel, C., Laville, J., Maurhofer, M., Schnider, U., et al. (2007). "Biocontrol of root diseases by Pseudomonas fluorescens CHA0: current concepts and experimental approaches," in Molecular Ecology of Rhizosphere Microorganisms (Weinheim: Wiley-VCH Verlag GmbH), 67-89. doi: 10.1002/9783527615810.ch6

Wenner, N., Maes, A., Cotado-Sampayo, M., and Lapouge, K. (2014). NrsZ: a novel, processed, nitrogen-dependent, small non-coding RNA that regulates Pseudomonas aeruginosa PAO1 virulence. Environ. Microbiol. 16, 1053-1068. doi: 10.1111/1462-2920.12272

Williams, D. R., Rowe, J. J., Romero, P., and Eagon, R. G. (1978). Denitrifying Pseudomonas aeruginosa: some parameters of growth and active transport. Appl. Environ. Microbiol. 36, 257-263.

Wolfinger, M. T., Fallmann, J., Eggenhofer, F., and Amman, F. (2015). ViennaNGS: a toolbox for building efficient next- generation sequencing analysis pipelines. F1000 Res. 4:50. doi: 10.12688/f1000research.6157.2

Wurtzel, O., Yoder-Himes, D. R., Han, K., Dandekar, A. A., Edelheit, S., Greenberg, E. P., et al. (2012). The single-nucleotide resolution transcriptome of Pseudomonas aeruginosa grown in body temperature. PLoS Pathog. 8:e1002945. doi: 10.1371/journal.ppat.1002945

Ye, R. W., Haas, D., Ka, J. O., Krishnapillai, V., Zimmermann, A., Baird, C., et al. (1995). Anaerobic activation of the entire denitrification pathway in Pseudomonas aeruginosa requires Anr, an analog of Fnr. J. Bacteriol. 177, 3606-3609. doi: 10.1128/jb.177.12.3606-3609.1995

Yoon, S. S., Hennigan, R. F., Hilliard, G. M., Ochsner, U. A., Parvatiyar, K., Kamani, M. C., et al. (2002). Pseudomonas aeruginosa anaerobic respiration in biofilms: relationships to cystic fibrosis pathogenesis. Dev. Cell 3, 593-603. doi: 10.1016/S1534-5807(02)00295-2

Yoon, S. S., Karabulut, A. C., Lipscomb, J. D., Hennigan, R. F., Lymar, S. V., Groce, S. L., et al. (2007). Two-pronged survival strategy for the major cystic fibrosis pathogen, Pseudomonas aeruginosa, lacking the capacity to 
degrade nitric oxide during anaerobic respiration. EMBO J. 26, 3662-3672. doi: 10.1038/sj.emboj.7601787

Zhang, Z., Schwartz, S., Wagner, L., and Miller, W. (2000). A greedy algorithm for aligning DNA sequences. J. Comput. Biol. 7, 203-214. doi: $10.1089 / 10665270050081478$

Zimmermann, A., Reimmann, C., Galimand, M., and Haas, D. (1991). Anaerobic growth and cyanide synthesis of Pseudomonas aeruginosa depend on anr, a regulatory gene homologous with fnr of Escherichia coli. Mol. Microbiol. 5, 1483-1490. doi: 10.1111/j.1365-2958.1991.tb00794.x

Zumft, W. G. (1997). Cell biology and molecular basis of denitrification. Microbiol. Mol. Biol. Rev. 61, 533-616.
Conflict of Interest Statement: The authors declare that the research was conducted in the absence of any commercial or financial relationships that could be construed as a potential conflict of interest.

Copyright (c) 2017 Tata, Amman, Pawar, Wolfinger, Weiss, Häussler and Bläsi. This is an open-access article distributed under the terms of the Creative Commons Attribution License (CC BY). The use, distribution or reproduction in other forums is permitted, provided the original author(s) or licensor are credited and that the original publication in this journal is cited, in accordance with accepted academic practice. No use, distribution or reproduction is permitted which does not comply with these terms. 